\title{
Preface to the Fifth French-Speaking Conference on Ontologies
}

\author{
Faiez Gargouri ${ }^{1}$
}

(C) Springer-Verlag Berlin Heidelberg 2016

This special issue of the Journal of Data Semantics includes the extended versions of five selected papers from the fifth French-Speaking Conference on Ontologies (Journées Francophones sur les Ontologies), which took place from November 14-16, 2014, in Hammamet, Tunisia. The papers were selected by taking into account the quality, significance and relevance of the results they present. All the extended papers went through an additional peer review process.

The first paper "Ontologies-Based Platform for Sociocultural Knowledge Management" by Papa Fary Diallo, Olivier Corby, Isabelle Mirbel, Moussa Lo and Seydina Ndiaye, presents a sociocultural platform aiming at persevering and capitalizing sociocultural events in Senegal. This platform relies on Semantic Web technologies. Authors discuss the two ontologies that they provided to support their platform: an upper-level sociocultural ontology (USCO) and a human time ontology (HuTO). To build the upper-level ontology, authors proposed a methodology based on the theory of Russian psychologist Lev Vygotsky called "Vygotskian Framework". They also present how this ontology can be matched in the Linked Open Data (LOD) cloud. On the other hand, they present the second ontology: HuTO, of which major contributions are (1) the modeling of nonconvex intervals (repetitive interval) like every Monday, (2) representation of deictic temporal expressions which form specific relations with time speech and (3) qualitative temporal notions which are temporal notions relative to a culture or a geographical position. Finally, the paper presents and discusses the platform designed on top of Semantic MediaWiki (SMW) to apply the authors' scientific contributions.

Faiez Gargouri

faiez.gargouri@isimsf.rnu.tn

1 Higher Institute of Computer Science and Multimedia, University of Sfax, BP 242, 3021 Sakiet Ezzeit Sfax, Tunisia
Indeed, the platform allows Senegalese communities to share and co-construct their sociocultural knowledge.

In the second paper, " $\tau$ OWL: A Systematic Approach to Temporal Versioning of Semantic Web Ontologies" by Abir Zekri, Zouhaier Brahmia, Fabio Grandi and Rafik Bouaziz, for a disciplined and systematic approach to the temporal management of Semantic Web ontologies, the authors propose the adoption of a framework called Temporal OWL 2 ( $\tau$ OWL) for a disciplined and systematic approach to the temporal management of Semantic Web documents. In a way similar to what happens in $\tau$ XSchema, $\tau$ OWL allows creating a temporal OWL 2 ontology from a conventional (i.e., nontemporal) OWL 2 ontology and a set of logical and physical annotations. Logical annotations identify which elements of the ontology can vary over time; physical annotations specify how the time-varying aspects are represented in the OWL 2 document. Using annotations to integrate temporal aspects in the traditional Semantic Web, the Temporal OWL 2 framework: (1) guarantees logical and physical data independence for temporal schemas and (2) provides a low-impact solution since it requires neither modifications of existing Semantic Web ontologies, nor extensions to the OWL 2 recommendation and Semantic Web standards. Moreover, since conventional schema and annotation documents could evolve over the time to respond to new applications' requirements,

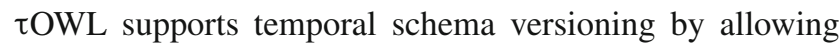
changing of these components and by keeping track of their evolution through conventional schema versions and annotation document versions, respectively. Two complete sets of operations are proposed for changing conventional schema and annotation documents; to complete the figure, a set of operations is also introduced for updating temporal schema which must be changed consequently each time one of the mentioned components evolves over time. Authors also 
present a prototype tool, named $\tau$ OWLManager, to show the feasibility of their approach.

In the third paper "A Core Ontology of Business Processes Based on DOLCE" by Mohamed Turki, Gilles Kassel, Inès Saad and Faïez Gargouri, the authors affirm that, new performance requirements, to adapt to the changing environment and to maintain a competitive advantage, have contributed, since the 1980's, to the emergence of new types of organizations focused on projects or processes. In fact, they notice that, to facilitate the implementation of this process view of organizations, many theoreticians and practitioners have proposed analysis and modeling frameworks, ontologies being considered as a relevant tool to conduct a "semantic analysis" of business processes. Indeed, approaches in this area are, however, based on ad hoc, (and often implicit) modeling principles, and so the proposed ontologies remain poor in terms of expressiveness. The objective of this paper is to analyze the ontological foundations of the business processes following a formal approach. Authors propose a core ontology of business processes by refining the DOLCE foundational ontology and supplementing Bottazzi and Ferrario's DOLCE-based formal ontology of organizations. This ontology comprises several modules to reflect both the "static" aspects of organizations and their behaviors, including intentional ones. The paper presents the contents of the ontology, the formal ontological tools reused for its design, and the various theories that the ontology is committed to.

The main field of interest of the paper "CSLAOnto: A Comprehensive Ontological SLA Model in Cloud Computing" by Taher Labidi, Achraf Mtibaa and Hayet Brabra, is cloud computing as an emerging topic in the information technology sector. The rapid evolution of this technology led to the emergence of new interaction models between its actors. However, the absence of a universally accepted format of the service level agreement (SLA) governing the relationships between the different actors of cloud increases its complexity. Also, the dynamic nature of the cloud could lead to severe SLA violations. This undesirable but currently unavoidable situation may lead to penalizing providers and forcing customers to evaluate their SLAs permanently. To deal with this problem, the authors propose, in this paper, a generic and semantically richer ontology-based model for SLA to improve its definition and its evaluation in cloud computing. Through this model, they have developed a prototype to validate their model and their monitoring process where SLA evaluation and guarantee actions triggering are automatically performed.

The last paper, "An Ontological Approach to Design Realtime Applications" by Wided Ben Abid, Mohamed Mhiri, Emna Bouazizi and Ahlem Rhayem, deals with using ontologies to help building databases. In fact, in these recent years, authors notice the emergence of several studies allowing a linkage between databases and ontologies to facilitate the design of databases. However, these studies do not address the needs of the advanced applications, because they do not integrate mechanisms that take into account the temporal characteristic. Also, the complexity of real-time applications is ever increasing which makes their design processes very difficult. For that purpose, a new vision will allow to fill in the limits of these studies and to suggest a method to conceptualize this kind of databases (real-time databases). This vision needs to define a new ontology to be integrated into these databases. This suggested ontology should be used to represent a domain in an explicit way without any ambiguity, to describe the most general categories and relations, to define the temporal information and to resolve the semantic conflicts. In this paper, authors present their solution to advance an ontological modeling for real-time applications.

I would sincerely like to thank the authors for their valuable contributions. 